\title{
Absent tibia-polydactyly-arachnoid cyst syndrome
}

INSERM

\section{Source}

INSERM. (1999). Orphanet: an online rare disease and orphan drug data base. Absent tibia-polydactyly-arachnoid cyst syndrome. ORPHA:3328

Tibia absent - polydactyly - arachnoid cyst syndrome is a very rare constellation of multiple anomalies, including absence or hypoplasia of the tibia. 